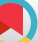

\title{
Etiologic Features of Respiratory Tract Infections Seen in Children with Primary Nephrotic Syndrome: A Cross-Sectional Retrospective Study
}

\author{
Xi-Lin Xiong ${ }^{1}$, Chun-Yu Yu ${ }^{1}$ and Mo Wang, ${ }^{1}$ \\ ${ }^{1}$ Department of Nephrology, Ministry of Education Key Laboratory of Child Development and Disorders, National Clinical Research Center for Child Health and Disorders, \\ China International Science and Technology Cooperation Base of Child Development and Critical Disorders, Children's Hospital of Chongqing Medical University, \\ Chongqing, PR China \\ "Corresponding author: Department of Nephrology, Ministry of Education Key Laboratory of Child Development and Disorders, National Clinical Research Center for Child \\ Health and Disorders, China International Science and Technology Cooperation Base of Child Development and Critical Disorders, Children's Hospital of Chongqing Medical \\ University, Chongqing, PR China. Email: wangmo_cqmu@163.com
}

Received 2019 November 02; Revised 2020 July 08; Accepted 2020 August 11.

\begin{abstract}
Background: Respiratory tract infections (RTI) are common complications of primary nephrotic syndrome (PNS) and influence the outcomes of PNS. The pathogenic spectrum and their susceptibility to antibiotics in RTI change over time, which increases the difficulty of treatment.

Objectives: To improve the curative effect of RTI in children with PNS, we conducted a cross-sectional retrospective study in 2740 children with PNS to study the etiologic features of RTI seen in PNS children.

Methods: Totally 2740 children with PNS hospitalized during 2010 - 2014 were enrolled in this study. Respiratory specimens were collected for the detection of pathogens using current laboratory diagnostic tests. The cases were divided into community-acquired respiratory tract infection (CARTI) and hospital-acquired respiratory tract infection (HARTI) as well as upper respiratory tract infection (URTI) and lower respiratory tract infection (LRTI) to analyse the etiologic features.

Results: Among 2162 cases of PNS with RTI, 2095 cases were CARTI (96.90\%), and 67 cases were HARTI (3.10\%). The most common pathogen in CARTI was Coxsackie virus, followed by Mycoplasma pneumoniae, respiratory syncytial virus, and Streptococcus pneumoniae, while the most common pathogen in HARTI also was Coxsackie virus, followed by respiratory syncytial virus, Mycoplasma pneumoniae, and Streptococcus pneumoniae. There was significant difference in the positive rates of Pseudomonas aeruginosa and Candida albicans between CARTI and HARTI. The distribution of pathogens differed between URTI and LRTI in children with PNS. The positive rates of most pathogens were lower in URTI than LRTI. Generally, the isolation rate of extended-spectrum $\beta$-lactamase (ESBL) producing bacteria was relatively low. In CARTI, the sensitive rates of Gram-negative ESBL producing bacteria to carbapenems, amoxicillin/clavulanate potassium, ampicillin/sulbactam, and ciprofloxacin were more than $90.00 \%$, while Gram-positive ESBL producing bacteria were sensitive to glycopeptides, linezolid, macrodantin, mupirocin-HL, and amoxicillin/clavulanate potassium.

Conclusions: In children with PNS, the etiology differed between CARTI and HARTI as well as URTI and LRTI. Therefore, it is necessary to differentiate these features for empirical therapy. If the pathogen is bacterial, amoxicillin/clavulanate potassium could be the first choice before sputum culture results are available.
\end{abstract}

Keywords: Children, Primary Nephrotic Syndrome, Respiratory Tract Infections, Etiology

\section{Background}

Primary nephrotic syndrome (PNS) is a disease characterized by the presence of nephrotic range proteinuria, clinical edema, hypoalbuminemia, and hyperlipidemia. It is one of the most common glomerular diseases in children, which accounts for 2 - 7 cases for every 100,000 children per year (1). Children with PNS have the secondary immunodeficiency showing the susceptibility to infection for their hypogammaglobulinemia and usage of glucocor- ticoid therapy. Respiratory tract infections (RTI) are common complications of PNS $(2,3)$. Infections not only reduce the efficacy of glucocorticoid treatment but also lead to prolonged duration and frequent recurrence in PNS (4, 5). The pathogenic spectrum and their susceptibility to antibiotics in RTI change over time, which increases the difficulty of anti-infection treatment. Therefore, prediction of the causative pathogens and their drug susceptibility before detecting results of respiratory pathogens in cases of 
PNS with RTI is needed to improve disease outcomes. There have been some researches on the etiology of RTI in China and abroad (6-8), but comprehensive studies on the etiology of RTI in children with PNS are scarce in the recent past.

\section{Objectives}

To improve the curative effect of PNS complicated with RTI, the clinical data of 2740 children with PNS who were inpatients in our hospital from 1.1.2010 - 12.13.2014 were analysed retrospectively in this study.

\section{Methods}

\subsection{Clinical Data}

2740 children diagnosed with PNS hospitalized in Children's Hospital of Chongqing Medical University during 01.01.2010 - 12.31.2014 were enrolled in this study. Diagnosis of PNS referred to the 2012 KDIGO clinical practice guideline for glomerulonephritis (9).

RTI were divided into community-acquired respiratory tract infection (CARTI) and hospital-acquired respiratory tract infections (HARTI) according to the time of onset or divided into upper respiratory tract infection (URTI) and lower respiratory tract infection (LRTI) according to the site of infection. Definition and criteria refer to $\mathrm{Zhu}$ Futang textbook of pediatrics (10). CARTI, RTI occur before or within 48 hours of hospitalization; HARTI, RTI occur after 48 hours of inpatient; URTI, acute infections occur above the larynx, including the nose and pharynx of the upper respiratory tract; LRTI, acute infections occur below the larynx.

For recruited patients, nasopharyngeal aspirates (NPAs) and blood samples were collected on hospital admission after obtaining informed consent for the detection of common respiratory viruses, bacteria, and other atypical pathogens using current laboratory diagnostic tests.

NPAs were preserved in virus transport medium immediately after collection, and stored at $-80^{\circ} \mathrm{C}$ prior to testing. Viral DNA and RNA were extracted from $200 \mathrm{~mL}$ of the NPA and eluted in $62 \mathrm{~mL}$ of AE Buffer by using QIAamp MinElute Virus Spin Kits (QIAGEN, Hilden, Germany). The complementary DNA sample was synthesized by using Super-Script First-Strand Synthesis system for reverse transcription polymerase chain reaction (RT-PCR) (Invitrogen, Camarillo, CA). All samples were screened by RT-PCR or PCR for common respiratory viruses using standard methods. Qualitative and semiquantitative cultures for bacteria were performed immediately using standard microbiological methods. Macroscopically distinct colonies of the samples were isolated in pure culture, and standard methods were used for identification, typing with sensitivity patterns.

Comparisons between different groups were performed using the chi-square test, and $\mathrm{P}<0.05$ was considered significant.

\section{Results}

\subsection{General Information}

Children with PNS complicated with RTI counted for $78.91 \%$ of our cases, totalling 2162 cases. The average age of the 2095 children with PNS and CARTI was $5.71 \pm 3.69$ years. The average age of the 67 children with PNS and HARTI was $5.8 \pm 4.05$ years. A total of 928 cases among the 2162 children with PNS and RTI underwent etiological examination; the examination rate was $42.92 \%$, and the positive rate was $56.57 \%$ (525 cases).

\subsection{The Pathogen Distribution of CARTI and HARTI in Cases with PNS}

In cases of PNS with CARTI, 579 cases underwent sputum culture, and 208 cases (35.92\%) were positive. A total of 270 strains of bacteria were isolated, among which there were 163 (60.37\%) Gram-negative strains and 107 (39.63\%) Gram-positive strains. Viruses, atypical pathogens and fungi were detected in 254, 117, and 12 of the specimens, respectively. The most common pathogen was Coxsackie virus, followed by Mycoplasma pneumoniae, Respiratory syncytial virus, Streptococcus pneumoniae, and Moraxella catarrhalis (Table 1 ).

In cases of PNS with HARTI, there were 15 positive specimens in 29 specimens for bacterial culture, with a positive rate of 51.72\%. 11 strains of Gram-negative bacteria (55.00\%) and 9 strains of Gram-positive bacteria (45.00\%) were isolated. Viruses, atypical pathogens and fungi were detected in 10,4 , and 2 of the specimens, respectively. The most common pathogen was Coxsackie virus, followed by Respiratory syncytial virus, Mycoplasma pneumoniae, Streptococcus pneumoniae, and Moraxella catarrhalis (Table 1).

The overall positive rate of bacteria was lower in CARTI (35.92\%) than HARTI (51.72\%), but the difference was not statistically significant $(\mathrm{P}>0.05)$. There was significant difference in the positive rates of Pseudomonas aeruginosa between 2 groups $(\mathrm{P}=0.02)$, which indicated that opportunistic infections are more common in HARTI. Besides, the positive rates of other pathogens were not significantly different between the two groups $(\mathrm{P}>0.05)$. 


\begin{tabular}{|c|c|c|c|c|c|}
\hline & \multicolumn{2}{|c|}{ CARTI } & \multicolumn{2}{|c|}{ HARTI } & \multirow{2}{*}{ P Value } \\
\hline & Strains (Strain) & Positive Rate, \% & Strains (Strain) & Positive Rate, \% & \\
\hline \multicolumn{6}{|l|}{ Bacteria } \\
\hline Moraxella catarrhalis & 55 & 9.5 & 4 & 13.79 & 0.659 \\
\hline Haemophilus parainfluenzae & 35 & 6.04 & 1 & 3.45 & 0.861 \\
\hline Haemophilus influenzae & 24 & 4.15 & 1 & 3.45 & 1.000 \\
\hline $\begin{array}{l}\text { Unidentified Gram-negative } \\
\text { bacteria }\end{array}$ & 21 & 3.63 & 2 & 6.9 & 0.688 \\
\hline Klebsiella pneumoniae & 12 & 2.07 & 0 & 0 & 1.000 \\
\hline Pseudomonas putida & 4 & 0.69 & 0 & 0 & 1.000 \\
\hline Acinetobacter baumannii & 4 & 0.69 & 0 & 0 & 1.000 \\
\hline Pseudomonas aeruginosa & 3 & 0.52 & 2 & 6.9 & 0.02 \\
\hline Escherichia coli & 1 & 0.17 & 1 & 3.45 & 0.093 \\
\hline Streptococcus pneumoniae & 65 & 11.23 & 5 & 17.24 & 0.489 \\
\hline $\begin{array}{l}\text { Unidentified Gram-positive } \\
\text { bacteria }\end{array}$ & 26 & 4.49 & 4 & 13.79 & 0.069 \\
\hline Staphylococcus aureus & 16 & 2.76 & 0 & 0 & 1.000 \\
\hline other & 4 & 0.68 & 0 & 0 & 1.000 \\
\hline \multicolumn{6}{|l|}{ Viruses } \\
\hline Coxsackie virus & 121 & 37 & 5 & 71.43 & 0.143 \\
\hline Respiratory syncytial virus & 79 & 17.25 & 6 & 30 & 0.246 \\
\hline Adenovirus & 46 & 8.2 & 0 & 0 & 0.438 \\
\hline Cytomegalovirus & 30 & 7.58 & 1 & 10 & 0.552 \\
\hline Parainfluenza virus 3 & 19 & 5.12 & 0 & 0 & 1.000 \\
\hline EB virus & 12 & 3.63 & 0 & 0 & 1.000 \\
\hline Influenza virus A & 12 & 3.23 & 1 & 6.25 & 0.427 \\
\hline Influenza virus B & 4 & 1.08 & 1 & 6.25 & 0.192 \\
\hline Parainfluenza virus 1 & 3 & 0.81 & 1 & 6.25 & 0.156 \\
\hline Herpes simplex virus & 1 & 0.86 & 0 & 0 & - \\
\hline \multicolumn{6}{|l|}{ Atypical pathogens } \\
\hline Mycoplasma pneumoniae & 112 & 23 & 4 & 28.57 & 0.868 \\
\hline Chlamydia & 5 & 2.04 & 0 & 0 & 1.000 \\
\hline \multicolumn{6}{|l|}{ Fungi } \\
\hline Candida albicans & 8 & 1.38 & 2 & 6.9 & 0.078 \\
\hline Unknown fungus & 3 & 0.52 & 0 & 0 & 1.000 \\
\hline Candida tropicalis & 1 & 0.17 & 0 & 0 & 1.000 \\
\hline
\end{tabular}

\subsection{The Pathogen Distribution of URTI and LRTI in Cases with} PNS

Among the cases of PNS with CARTI, there were 1317 cases of URTI (62.86\%) and 778 cases of LRTI (37.14\%). In URTI, the most common pathogen was Coxsackie virus, followed by Mycoplasma pneumoniae, Respiratory syncytial virus, Streptococcus pneumoniae, and Adenovirus (Figure 1).
In LRTI, the most common pathogen was Coxsackie virus, followed by Mycoplasma pneumoniae, Respiratory syncytial virus, Streptococcus pneumoniae, and Moraxella catarrhalis (Figure 1).

The distribution of the respiratory pathogens varied batween URTI and LRTI (Figure 1). The positive rate of Haemophilus influenzae was considerably lower in URTI 
A

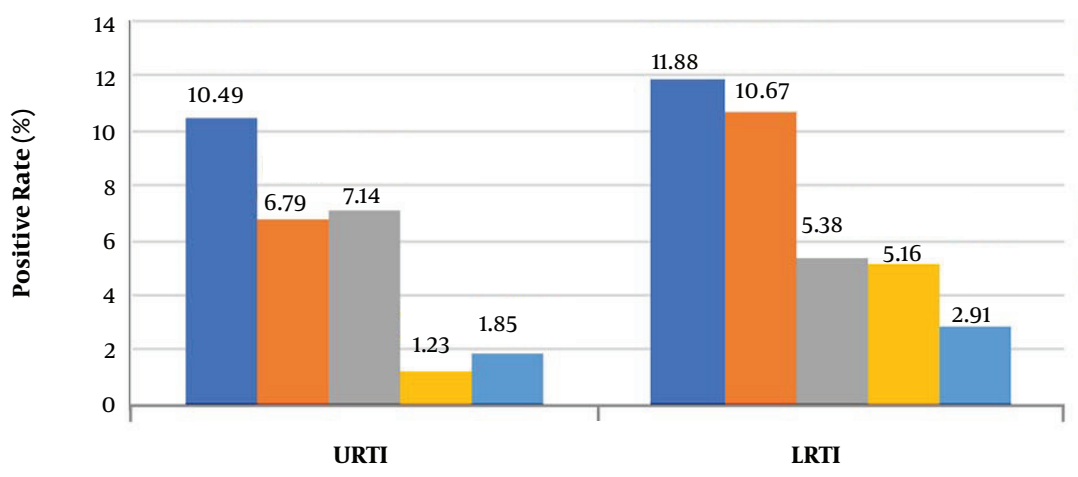

B

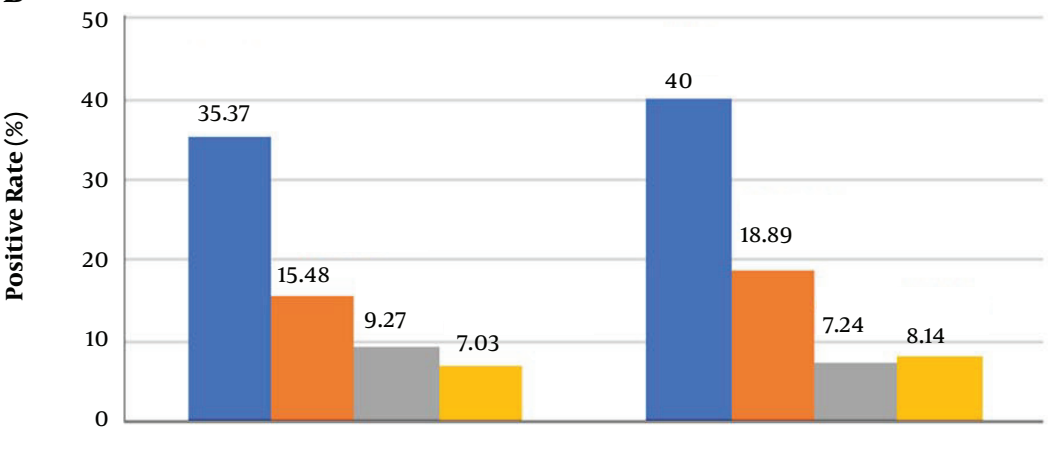

URTI

C

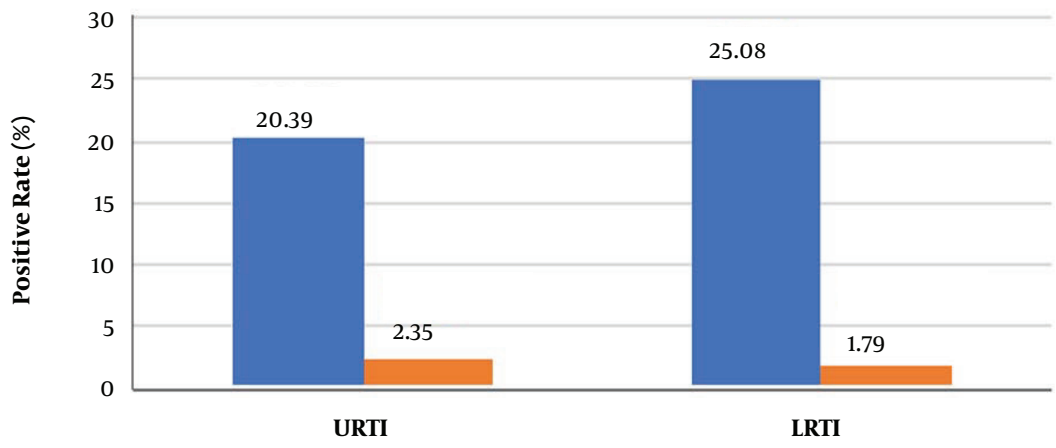

Streptococcus Pneumoniae

Moraxella Catarrhalis

Haemophilus Parainfluenzae

Haemophilus Iufluenzae

Staphylococcus Aureus
Coxsackie Virus

Respiratory Syncytial Virus

Adenovirus

Cytomegalovirus
Mycoplasma Pneumoniae

Chlamydia

Figure 1. Positive rates of common pathogens in URTI and LRTI. A, B, and C, indicate the positive rates of common bacteria, viruses and atypical pathogens in URTI and LRTI, respectively.

than LRTI, which was significantly different $(\mathrm{P}=0.031)$. The positive rates of other pathogens shown in Figure1 differed between 2 groups, but the results weren't statistically significant $(\mathrm{P}>0.05)$.

\subsection{Analysis of Antibiotic Resistance of Bacteria}

In cases of PNS with CARTI, 49 strains of extendedspectrum $\beta$-lactamase (ESBL) producing bacteria (18.15\%) were isolated among 270 strains of bacteria, including 39 strains of Gram-negative bacteria and 10 strains of Gram- 
positive bacteria. In cases of PNS with HARTI, 3 strains of ESBL producing bacteria (15.00\%) were isolated among 20 strains of bacteria and all were Gram-negative bacteria. The most common ESBL producing bacterium was Moraxella catarrhalis. Table 2 compares different ESBL producing strains and their positive rates between CARTI and HARTI.

In cases of PNS with CARTI, the sensitive rates of Gram-negative ESBL producing bacteria to carbapenems, gentamicin, levofloxacin, amoxicillin/clavulanate potassium, ampicillin/sulbactam, and ciprofloxacin were more than $90.00 \%$ (Table 3). Gram-positive ESBL producing bacteria were highly sensitive to glycopeptides, linezolid, quinolones, rifampicin, Macrodantin, mupirocinHL, quinupristin/dalfopristin, and amoxicillin/clavulanate potassium with a sensitive rate more than $80.00 \%$ (Table 4). There was no statistical significance to analyse the antibiotic susceptibility and resistance of ESBL producing bacteria in HARTI because of the small sample size.

\section{Discussion}

Owing to the substantial loss of protein in urine, the immunoglobulin of children with PNS stays at a very low level. Infection could occur in every system. RTI is one of the most common complications and has become a significant cause of relapse and death. In this study, the incidence rate of PNS complicated with RTI was 78.91\%, which was higher than the incidence rate (72\%) in Saudi Arabia reported by Alfakeekh et al. (1).

Our data showed that preschool and school-age children were likely to develop an RTI, while Wei et al. (2) reported that children under 10 were at a higher risk than children older than 10 in Taiwan. Different susceptible ages might vary in different times and regions. The main infection site of PNS complicated with RTI was the upper respiratory tract, which was similar to the findings reported in Japan (11). Therefore, we need to strengthen the management and screening of RTI in preschool and school-age children with PNS.

This study showed that the pathogen spectrum differed between cases of PNS with CARTI and with HARTI. The three most common bacteria in cases of PNS with CARTI were Streptococcus pneumoniae, Moraxella catarrhalis, and Haemophilus parainfluenzae. In the same period, the three most common bacteria in other cases of CARTI without PNS in local areas were Haemophilus parainfluenzae, Streptococcus pneumoniae and Moraxella catarrhalis (6), which was similar to our study. In addition, Streptococcus pneumoniae was the most common bacterium in cases of PNS with RTI in our centre, which was different from the main bacterium in hemodialysis patients reported by Gupta V (12),
Staphylococcus aureus. This finding indicated that children with different kidney diseases developed RTI caused by different pathogens.

The most common virus in cases of PNS with CARTI was Coxsackie virus, followed by respiratory syncytial virus and adenovirus, while the most common virus in cases of PNS with HARTI was Coxsackie virus, followed by respiratory syncytial virus and cytomegalovirus. In the same period, the most frequently detected viruses in other cases of CARTI without PNS in local areas were respiratory syncytial virus, parainfluenza virus, and influenza virus (6), which was different to our study. The constitution of viruses in cases of PNS with RTI was different from the constitution of viruses in cases of RTI in children in eastern and southern China $(13,14)$. Our data showed that Coxsackie virus was the most common virus in cases of PNS with RTI, which was different from the respiratory syncytial virus suggested by foreign literature $(15,16)$. We considered that the main cause was age. Most of the children in our study were preschool- or school-age children. Coxsackie virus infection is likely to occur in children younger than 6 , and respiratory syncytial virus infection is likely to occur in infants. Cytomegalovirus is one of the most common pathogens in infectious disease of infants and toddlers. The main target organ is the liver, but it can also lead to pulmonary infection. According to Doan et al. (17), cases of cytomegalovirus pneumonia often occur in infants under 3 months, indicating that cytomegalovirus is not the main pathogen in older children with RTI. Cytomegalovirus pneumonia should be diagnosed by corresponding signs in physical examination and CMV DNA quantitation in bronchoalveolar lavage fluid (18). However, cytomegalovirus in our patients was not detected in bronchoalveolar lavage fluid, it was detected by ELISA or PCR analysis of blood samples. We did not target the respiratory system to detect cytomegalovirus, so the results were not specific enough. Therefore, cytomegalovirus was not regarded as the main virus in cases of PNS with RTI in this study.

In this study, Candida albicans was the main fungus in cases of PNS with RTI, which was a similar result to that reported by Liu P(7).

Mycoplasma pneumoniae is a common pathogen in cases of CARTI in children. Previous studies show that the detected rate of Mycoplasma pneumoniae ranges from $8 \%$ $35 \%$ in $\operatorname{CARTI}(7,8,19)$. In our data, the infection rate of $M y$ coplasma pneumoniae was $23 \%$ in cases of PNS with CARTI and $28.57 \%$ in cases of PNS with HARTI, and there were no significant differences between them $(\mathrm{P}>0.05)$. Therefore, we suggest that the detection of Mycoplasma pneumoniae should be a routine test in cases of PNS with RTI.

According to this study, the distribution of bacteria differed between cases of URTI and LRTI in PNS with RTI. There- 


\begin{tabular}{|c|c|c|c|c|c|}
\hline \multirow{2}{*}{ Bacteria } & \multicolumn{2}{|l|}{ CARTI } & \multicolumn{2}{|l|}{ HARTI } & \multirow{2}{*}{ P Value } \\
\hline & Strains (Strain) & Rate, \% & Strains (Strain) & Rate, \% & \\
\hline Gram-negative bacteria & 39 & 23.93 & 3 & 27.27 & 1.000 \\
\hline Moraxella catarrhalis & 29 & 52.73 & 1 & 25 & 0.580 \\
\hline Haemophilus influenzae & 7 & 29.17 & 1 & 100 & 0.320 \\
\hline Enterobacter aerogenes & 1 & 100 & 0 & 0 & - \\
\hline Escherichia coli & 1 & 100 & 1 & 100 & 1.000 \\
\hline Gram-positive bacteria & 10 & 9.35 & 0 & 0 & 1.000 \\
\hline Staphylococcus aureus & 10 & 62.5 & 0 & 0 & - \\
\hline
\end{tabular}

Table 3. Susceptibility and Resistance of Gram-Negative ESBL-Carrying Bacteria in CARTI

\begin{tabular}{|c|c|c|}
\hline Antibiotics & Susceptible (Ratio \%) & Resistant (Ratio \%) \\
\hline Imipenem & 100 & 0 \\
\hline Meropenem & 100 & 0 \\
\hline Gentamicin & 100 & 0 \\
\hline Levofloxacin & 100 & 0 \\
\hline Ceftriaxone & 100 & 0 \\
\hline Rifampicin & 97.3 & 0 \\
\hline Ceftazidime & 94.74 & 5.26 \\
\hline Amoxicillin/clavulanate & 92.31 & 7.69 \\
\hline Ampicillin/sulbactam & 92.31 & 7.69 \\
\hline Ciprofloxacin & 92.31 & 7.69 \\
\hline Cefotaxime & 92.31 & 7.69 \\
\hline Cefuroxime & 91.89 & 5.41 \\
\hline Cefprozil & 82.35 & 17.65 \\
\hline Cefaclor & 50 & 28.57 \\
\hline Cefepime & 50 & 50 \\
\hline Chloramphenicol & 82.05 & 15.38 \\
\hline Azithromycin & 77.14 & 22.86 \\
\hline Aztreonam & 69.23 & 30.77 \\
\hline Tetracycline & 52.63 & 44.74 \\
\hline Piperacillin/sulbactam & 50 & 0 \\
\hline Piperacillin & 0 & 100 \\
\hline Ampicillin & 0 & 97.44 \\
\hline $\begin{array}{l}\text { Trimethoprim/ } \\
\text { Sulfamethoxazole }\end{array}$ & 33.33 & 66.67 \\
\hline Cefazolin & 0 & 100 \\
\hline
\end{tabular}

fore, we need to differentiate the location of RTI for empirical therapy in cases of PNS with RTI.

In this study, the ESBL positive rate of Gram-negative bacteria in cases of PNS with CARTI was $23.93 \%$, which
Table 4. Susceptibility and Resistance of Gram-Positive ESBL-Carrying Bacteria in CARTI

\begin{tabular}{|lcc}
\hline Antibiotics & Susceptible $($ Ratio $\%)$ & Resistant (Ratio $\%)$ \\
\hline Vancomycin & 100 & 0 \\
\hline Linezolid & 100 & 0 \\
\hline Teicoplanin & 100 & 0 \\
\hline Amikacin & 100 & 0 \\
\hline Ciprofloxacin & 100 & 0 \\
\hline Rifampicin & 100 & 0 \\
\hline Macrodantin & 100 & 0 \\
\hline Mupirocin-HL & 100 & 0 \\
\hline Quinupristin/dalfopristin & 100 & 0 \\
\hline Amoxicillin/clavulanate & 80 & 20 \\
\hline Oxacillin & 77.78 & 22.22 \\
\hline Penicillin & 0 & 100 \\
\hline Ampicillin & 0 & 100 \\
\hline Trimethoprim/sulfametho & 70 & 30 \\
\hline Trimethoprim & 70 & 20 \\
\hline Tetracycline & 62.5 & 25 \\
\hline Gentamicin & 60 & 40 \\
\hline Tobramycin & 60 & 40 \\
\hline Clindamycin & 30 & 70 \\
\hline Erythromycin & 30 & 70 \\
\hline Cefoxitin & 20 & 0 \\
\hline Fusidic acid & 20 & 0 \\
\hline Mupirocin & 20 & 0 \\
\hline & & 0 \\
\hline
\end{tabular}

was obviously higher than that of Gram-negative bacteria (9.35\%). Because the production of ESBL is mainly the resistance mechanism of Gram-negative bacteria.

In cases of PNS with HARTI, the ESBL positive rate of Gram-negative bacteria was $27.27 \%$, which was not significantly different from that in cases of PNS with CARTI (P > 
0.05). No ESBL producing bacteria were isolated among 9 strains of Gram-positive bacteria, which might be related to the small sample size of cases of HARTI.

According to the antibiotic susceptibility tests of drug-resistant strains, Gram-negative ESBL producing bacteria in cases of PNS with CARTI were highly sensitive to carbapenems, gentamicin, quinolones, rifampicin, ampicillin/sulbactam, and amoxicillin/clavulanate potassium. With a view to drug toxicity and price, amoxicillin/clavulanate potassium and ampicillin/sulbactam can be the first-line drugs for Gram-negative ESBL producing bacteria in cases of PNS with CARTI. Gram-positive ESBL producing bacteria were relatively sensitive to amoxicillin/clavulanate potassium. However, some $\beta$-lactamase inhibitors cannot completely inhibit ESBLs. If amoxicillin/clavulanate potassium and ampicillin/sulbactam are ineffective, we can choose carbapenems (20).

This study has some limitations. First, some common respiratory pathogens, such as human rhinovirus, human bocavirus, and human coronavirus, were not included in our study, which may lead to underestimation of the viral burden. Second, our findings can only be representative of children with PNS in local area, our findings may not be generalizable to other regions. Finally, we studied only hospitalized children, and a study of outpatients might have produced different results.

\subsection{Conclusions}

In conclusion, CARTI was more common than HARTI in cases of PNS with RTI, and URTI was more common than LRTI. Children of preschool and school age with PNS were more likely than younger or older children to develop an RTI. In children with PNS, the etiology differed between CARTI and HARTI as well as URTI and LRTI. Therefore, it is necessary to differentiate these features for empirical therapy. And we need to complete pathogen detection without delay. If the pathogen was bacterial, amoxicillin/clavulanate potassium could be the first choice before sputum culture results are available. We need to control infection as soon as possible to reduce the recurrence rate and improve the prognosis of children with PNS.

\section{Footnotes}

Authors' Contribution: Study concept and design: XX, and MW. Analysis and interpretation of data: XX and CY. Drafting of the manuscript: XX. Critical revision of the manuscript for important intellectual content: XX, CY, and MW. Statistical analysis: CY.

Conflict of Interests: The authors declare there is no conflicts of interest regarding the publication of this paper.
Ethical Approval: This study was performed with the approval of the Ethical Committees of Childrens Hospital of Chongqing Medical University.

Funding/Support: This study was supported in part by a teaching and research scholarship from the Chongqing Medical University.

Informed Consent: Obtained informed consent for the detection of common respiratory viruses, bacteria, and other atypical pathogens using current laboratory diagnostic tests.

\section{References}

1. Alfakeekh K, Azar M, Sowailmi BA, Alsulaiman S, Makdob SA, Omair $A$, et al. Immunosuppressive burden and risk factors of infection in primary childhood nephrotic syndrome. J Infect Public Health. 2019;12(1):90-4. doi:10.1016/j.jiph.2018.09.006. [PubMed: 30279098].

2. Wei CC, Yu IW, Lin HW, Tsai AC. Occurrence of infection among children with nephrotic syndrome during hospitalizations. Nephrology (Carlton). 2012;17(8):681-8. doi: 10.1111/j.1440-1797.2012.01650.x. [PubMed: 22882426].

3. Moorani KN, Khan KM, Ramzan A. Infections in children with nephrotic syndrome. J Coll Physicians Surg Pak. 2003;13(6):337-9.

4. Kim MY, Cho MH, Kim JH, Ahn YH, Choi HJ, Ha IS, et al. Acute kidney injury in childhood-onset nephrotic syndrome: Incidence and risk factors in hospitalized patients. Kidney Res Clin Pract. 2018;37(4):347-55. doi: 10.23876/j.krcp.18.0098. [PubMed: 30619690]. [PubMed Central: PMC6312784].

5. Dakshayani B, Lakshmanna M, Premalatha R. Predictors of frequent relapsing and steroid-dependent nephrotic syndrome in children. Turk Pediatri Ars. 2018;53(1):24-30. doi: 10.5152/TurkPediatriArs.2018.5749. [PubMed: 30083071]. [PubMed Central: PMC6070226].

6. Wei L, Liu W, Zhang XA, Liu EM, Wo Y, Cowling BJ, et al. Detection of viral and bacterial pathogens in hospitalized children with acute respiratory illnesses, Chongqing, 2009-2013. Medicine (Baltimore). 2015;94(16). e742. doi: 10.1097/MD.0000000000000742. [PubMed: 25906103]. [PubMed Central: PMC4602679].

7. Liu P, Xu M, He L, Su L, Wang A, Fu P, et al. Epidemiology of respiratory pathogens in children with lower respiratory tract infections in shanghai, china, from 2013 to 2015.Jpn JInfect Dis. 2018;71(1):39-44. doi: 10.7883/yoken.JJID.2017.323. [PubMed: 29279451].

8. Tsolia MN, Psarras S, Bossios A, Audi H, Paldanius M, Gourgiotis D, et al. Etiology of community-acquired pneumonia in hospitalized schoolage children: evidence for high prevalence of viral infections. Clin Infect Dis. 2004;39(5):681-6. doi: 10.1086/422996. [PubMed: 15356783]. [PubMed Central: PMC7107828].

9. Cattran DC, Feehally J, Cook HT, Liu ZH, Fervenza FC, Mezzano SA, et al. Kidney disease: improving global outcomes (KDIGO) glomerulonephritis work group. KDIGO clinical practice guideline for glomerulonephritis. Kidney Int Suppl. 2012;2(2):139-274.

10. Hu YM, Jiang ZF. Zhu futang textbook of pediatrics. 2002.

11. Ogi M, Yokoyama H, Tomosugi N, Hisada Y, Ohta S, Takaeda M, et al. Risk factors for infection and immunoglobulin replacement therapy in adult nephrotic syndrome. Am J Kidney Dis. 1994;24(3):427-36. doi: 10.1016/s0272-6386(12)80899-7.

12. Gupta V, Yassin MH. Infection and hemodialysis access: an updated review. Infect Disord Drug Targets. 2013;13(3):196-205. doi: 10.2174/1871526511313030008. [PubMed: 24001331].

13. Ge X, Guo Y, Chen J, Hu R, Feng X. Epidemiology and Seasonality of Respiratory Viruses Detected from Children with Respiratory Tract Infections in Wuxi, East China. Med Sci Monit. 2018;24:1856-62. 
doi: 10.12659/msm.908483. [PubMed: 29599424]. [PubMed Central: PMC5892462].

14. Zhang D, He Z, Xu L, Zhu X, Wu J, Wen W, et al. Epidemiology characteristics of respiratory viruses found in children and adults with respiratory tract infections in southern China. Int J Infect Dis. 2014;25:159-64. doi: 10.1016/j.ijid.2014.02.019. [PubMed: 24927663]. [PubMed Central: PMC7110535].

15. Hatipoğlu N, Somer A, Badur S, Ünüvar E, Akçay-Ciblak M, Yekeler E, et al.Viral etiology in hospitalized children with acute lower respiratory tract infection. Turk J Pediatr. 2011;53(5):508-16.

16. Khor CS, Sam IC, Hooi PS, Quek KF, Chan YF. Epidemiology and seasonality of respiratory viral infections in hospitalized children in Kuala Lumpur, Malaysia: a retrospective study of 27 years. BMCPediatr. 2012;12:32. doi: 10.1186/1471-2431-12-32. [PubMed: 22429933]. [PubMed Central: PMC3337250].

17. Doan TT, Phung TT, Pham HV, Pham SH, Nguyen LT. Effect of ganci- clovir for the treatment of severe cytomegalovirus-associated pneumonia in children without a specific immunocompromised state. BMC Infect Dis. 2013;13:424. doi: 10.1186/1471-2334-13-424. [PubMed 24010978]. [PubMed Central: PMC4015554].

18. Boeckh M, Stevens-Ayers T, Travi G, Huang ML, Cheng GS, Xie H, et al Cytomegalovirus (CMV) DNA Quantitation in Bronchoalveolar Lavage Fluid From Hematopoietic Stem Cell Transplant Recipients With CMV Pneumonia.JInfect Dis. 2017;215(10):1514-22. doi:10.1093/infdis/jix048. [PubMed: 28181657]. [PubMed Central: PMC5461426].

19. Jain S, Williams DJ, Arnold SR, Ampofo K, Bramley AM, Reed C, et al. Community-acquired pneumonia requiring hospitalization among U.S. children. N Engl J Med. 2015;372(9):835-45. doi: 10.1056/NEJMoa1405870. [PubMed: 25714161]. [PubMed Central: PMC4697461].

20. Mathur P, Kapil A. das b, dhawan b. Prevalence of extended spectrum beta lactamase producing gram negative bacteria in a tertiary care hospital. Indian J Med Res. 2002;115:153-7. 